\title{
BER Performance and Data Throughput of Sinusoidal Template- Based Impulse-Radio Ultra Wideband Receivers for Wireless Body Area Networks
}

\author{
Mohamad Abou El-Nasr and Heba Shaban \\ Arab Academy for Science, Technology and Maritime Transport (AASTMT), Alexandria, Egypt
}

Received 2013-01-15; Revised 2013-01-16; Accepted 3013-05-30

\begin{abstract}
Windowed sinusoidal template-based correlator receivers are good candidates for high-data rate low-power Ultra Wideband (UWB) systems. Their performance approaches the performance of optimal detectors and provides low-power consumption. However, the ultra short pulses of UWB technology makes it sensitive to timing-errors. In this paper, the Bit-Error-Rate (BER) performance and maximum allowable data throughput of Equally-Correlated Pulse Position Modulation (EC-PPM) modulation scheme in the presence of timingjitter are studied and compared for low-power sinusoidal template based correlator receivers. Additive White Gaussian Noise (AWGN), Nakagami-m fading, industrially accepted on-body IEEE 802.15.6a and actual onbody channels are considered. Numerical results show that the maximum SNR penalty when using sinusoidal templates is $\approx 2 \mathrm{~dB}$ for the same BER and the corresponding data-throughput degradation is $5 \mathrm{Mbps}$.
\end{abstract}

Keywords: Error Analysis, Low-Power Receivers, Timing-Jitter, Ultra-Wideband (UWB), Wireless Body Area Networks (WBANs)

\section{INTRODUCTION}

Impulse-Radio Ultra Wideband (IR-UWB) technology has the potential for very high data-rate, lowcost and low-complexity transceiver design. In addition, IR-UWB systems operate at very low-power levels, $41.3 \mathrm{dBm} / \mathrm{MHz}$, due to their high fractional bandwidths. Such advantages make it an attractive candidate for emerging high data-rate applications of Wireless Body Area Network (WBANs). However, due to the very short durations of UWB pulses, they require high sensitivity against timing-errors (Guvenc and Arslan, 2004; Lazaro et al., 2010; Shaban et al., 2011).

Another important issue associated with the application of IR-UWB for high data-rate applications of on-body WBANs is the power consumption of the receiver. Typically, non-coherent UWB receivers, such as Transmitted Reference (TR) and Energy Detection (ED) structures are ideal for low-power applications of UWB systems. However, their performance is highly suboptimal as compared to coherent detectors. Recently, low-power suboptimal template-based correlator receivers have been proposed as an alternate candidate that provides an approaching performance to optimal detectors with low-power consumption (Shaban et al., 2009; 2010).

In this paper, we study the effect of timing-jitter on the Bit-Error-Rate (BER) performance and data throughput of suboptimal sinusoidal template-based correlator receivers for WBAN applications. Specifically, we study the effect of timing-jitter on the BER performance of sinusoidal template-based correlator receivers for Equally-Correlated Pulse Position Modulation (EC-PPM) scheme in AdditiveAlexandria, Egypt 
White Gaussian Noise (AWGN) and independent and correlated Nakagami-m channels. We also study the corresponding maximum allowable data throughput, which is dependent on the BER. Moreover, we provide numerical results based on semi-analytic simulations in the IEEE 802.15.6a on-body CM\#3 UWB channel model (Yazdandoost and Pour, 2010) and actual on-body measurements for examining the performance of the investigated scheme for WBANs.

The organization of the paper is as follows. Section 2 introduces the optimal and suboptimal template pulses and their controlling design parameters. Then, Section 3 studies the effect of timing-jitter on the theoretical BER and data throughput of EC-PPM scheme in AWGN and Nakagami-m fading channels. Section 4 describes the actual measurement setup and provides numerical results based on simulations and actual measurements. Finally, conclusions are given in Section 5.

\subsection{Template Pulse}

The $n$-th order Gaussian pulse $\omega_{0}(\mathrm{t})$ has the form (Shaban et al., 2010) Equation (1):

$\omega_{\mathrm{n}}(\mathrm{t})=\frac{\mathrm{d}^{(\mathrm{n})}}{d \mathrm{t}^{\mathrm{n}}}\left(\mathrm{e}^{-2 \pi\left(\frac{\mathrm{t}}{\tau_{\mathrm{p}}}\right)^{2}}\right)$

where, $\tau_{\mathrm{p}}$ is equal to half the pulse width $\mathrm{T}_{\mathrm{p}}$. Assuming a correlation receiver, the optimal template $\mathrm{v}(\mathrm{t})$ should be matched to the received pulse $p(t)=\omega_{n}(t)$, where the pulse parameters are chosen to meet a specified Federal Communication Commission (FCC) system's allowable emission limits. When using a suboptimal sinusoidal template, the pulse width should be chosen to maximize the output Signal-to-Noise-Ratio (SNR).

For a sinusoidal template $\mathrm{v}(\mathrm{t})=\cos \left(\omega_{\mathrm{c}}(\mathrm{t})\right)$ for $\mathrm{a}$ window-length $\mathrm{T}$ and carrier frequency $\omega_{\mathrm{c}}$, the output SNR of the correlator receiver is given by (Shaban, 2010; Sangyoub, 2002) Equation (2):

$$
\mathrm{SNR}=\frac{\mathrm{E}_{\mathrm{b}}}{\mathrm{N}_{0}} \frac{\mathrm{R}_{\mathrm{pv}}^{2}\left(\tau_{\mathrm{e}}\right)}{\mathrm{R}_{\mathrm{vv}}(0)}
$$

where, $\mathrm{E}_{\mathrm{b}}$ is the bit energy, $\mathrm{N}_{0}$ is the noise PowerSpectral-Density (PSD), $\mathrm{R}_{\mathrm{pv}}($. ) is the normalized crosscorrelation of the received pulse and the template waveform, $\tau_{\mathrm{e}}$ is the timing error and $\mathrm{R}_{\mathrm{w}}($.$) is the$ normalized auto-correlation of the template pulse.

\subsection{Performance in AWGN and Multipath Channels}

In this section, we provide analytical analysis of the BER performance of suboptimal-template based coherent correlator receivers in AWGN and independent and correlated Nakagami-m channels assuming EC-PPM modulation.

\subsection{Performance in AWGN Channel}

Considering binary EC-PPM, with a transmitted pulse $\mathrm{p}(\mathrm{t})$, the transmitted signal is composed of $\mathrm{N}_{\mathrm{s}}$ time shifted pulses with $2 \leq \mathrm{M}<\mathrm{N}_{\mathrm{s}}$ and each signal is identified by a sequence of cyclic shifts of an $m$ sequence of length $\mathrm{N}_{\mathrm{s}}$ (Shaban et al., 2009). The optimal template is $(\mathrm{Hu}$ and Bueaulieu, 2005) Equation (3):

$$
v(t)=p(t)-p(t-\delta)
$$

where, $\delta$ is the PPM modulation parameter. In the case of the optimum receiver, the BER can be minimized by choosing $\delta$ to minimize the autocorrelation $\mathrm{R}_{\mathrm{pp}}(\delta)$ (Remirez-Mireles, 2001) Equation (4):

$\delta_{\mathrm{opt}}=\arg \left(\min _{\delta} R_{\mathrm{pp}}(\delta)\right)$

where, $\arg ($.$) is the argument function. For M-ary EC-$ PPM, the transmitted signal is composed of $\mathrm{N}_{\mathrm{s}}$ timeshifted pulses with $2 \leq \mathrm{M}<\mathrm{N}_{\mathrm{s}}$, where each signal is identified by a sequence of cyclic shifts of an m-sequence of length $\mathrm{N}_{\mathrm{s}}$ [10]. The union bound on the bit error probability of EC M-ary PPM assuming an optimum receiver in the presence of timing-error is Equation (5):

$\mathrm{U}_{\mathrm{BPb}}=\frac{\mathrm{M}}{2} \mathrm{Q}\left(\sqrt{\frac{\mathrm{E}_{\mathrm{b}}}{2 \mathrm{~N}_{0}} \frac{\left(\mathrm{R}_{\mathrm{pp}}\left(\tau_{\mathrm{e}}\right)-\mathrm{R}_{\mathrm{pp}}\left(\delta_{\mathrm{opt}}-\tau_{\mathrm{e}}\right)\right)^{2}}{\mathrm{R}_{\mathrm{pp}}(0)-\mathrm{R}_{\mathrm{pp}}\left(\delta_{\mathrm{opt}}\right)}}\right)$

where, Q(.) is the Gaussian tail function (Remirez-Mireles, 2001; Simon and Alouni, 2000) and the normalized pulse autocorrelation $\mathrm{R}_{\mathrm{pp} \min } \square \mathrm{R}_{\mathrm{pp}}\left(\delta_{\mathrm{opt}}\right)$.

The normalized cross-correlation function of the received pulse and sinusoidal template can be calculated as (Sangyoub, 2002) Equation (6):

$$
R_{p v}(\tau)=\frac{1}{\sqrt{E_{p}} \sqrt{E_{v}}} \int_{-T / 2}^{T / 2} p(t) \cos \left(\omega_{c}(t-\tau)\right) d t
$$


where, $E_{v}$ is the template pulse energy and $E_{p}$ is the received pulse energy.

To minimize BER, the value of $\delta$ should be chosen to minimize the correlation $\mathrm{R}_{\text {pvmin }}\left(\delta_{\text {opt }}\right)$. In addition, at the receiver a sample time $\mu$ is chosen to maximize the correlation between the sub-optimal template and the generated pulse Equation (7):

$\mu_{\mathrm{opt}}=\arg \left(\max _{\mu} R_{\mathrm{pv}}(\mu)\right)$

with $\mathrm{R}_{\mathrm{pvmix}}=\mathrm{R}_{\mathrm{pv}}\left(\mu_{\mathrm{opt}}\right)$, the union bound on the bit error probability for equally-correlated signals in the presence of timing-jitter is calculated as Equation (8):

$$
\mathrm{U}_{\mathrm{BPb}}=\frac{\mathrm{M}}{2} \mathrm{Q}\left(\sqrt{\frac{\mathrm{E}_{\mathrm{b}}}{2 \mathrm{~N}_{0}} \frac{\left(\mathrm{R}_{\mathrm{pv}}\left(\tau_{\mathrm{e}}\right)-\mathrm{R}_{\mathrm{pv}}\left(\mu_{\mathrm{opt}}-\tau_{\mathrm{e}}\right)\right)^{2}}{\left(\mathrm{R}_{\mathrm{pv}}(0)-\mathrm{R}_{\mathrm{pv}}\left(\mu_{\mathrm{opt}}\right)\right)}}\right)
$$

\subsection{Performance in Dense Multipath Channel}

The BER of low-complexity Partial-Rake (PRake) receivers (Quek and Win, 2005), assuming EC-PPM modulation and optimal templates in the presence of timing-jitter in terms of the Moment Generating Function (MGF), $\mathrm{M}_{\eta}$, over a Nakagami-m channel with uniform Power Delay Profile (PDP) and $\mathrm{L}_{\mathrm{p}}$ independent identically distributed (i.i.d.) paths is Equation (9):

$$
\mathrm{P}_{\mathrm{b}, \text { PRake }}=\frac{1}{\pi} \int_{0}^{\pi / 2}\left(\mathrm{M}_{\eta_{1}}\left(-\frac{\left(\mathrm{R}_{\mathrm{pp}}\left(\tau_{\mathrm{e}}\right)-\mathrm{R}_{\mathrm{pp}}\left(\delta_{\mathrm{opt}}-\tau_{\mathrm{e}}\right)\right)^{2}}{\left(\mathrm{R}_{\mathrm{pp}}(0)-\mathrm{R}_{\mathrm{pp}}\left(\delta_{\mathrm{opt}}\right)\right) 4 \mathrm{~m} \sin ^{2} \theta}\right)\right)^{\mathrm{L}_{\mathrm{p}}} \mathrm{d} \theta
$$

where, $\bar{\eta}=\mathrm{E}_{\mathrm{s}} / \mathrm{LN}_{0}$. Ideal Rake (ARake) receivers capture all the energy in all $\mathrm{L}$ paths, i.e., $\mathrm{L}_{\mathrm{p}}=\mathrm{L}$ (Quek and Win, 2005). Substituting with the MGF $\mathrm{M}_{\bar{\eta}}(\mathrm{s})=\left(1-\frac{\mathrm{s} \bar{\eta}}{\mathrm{m}}\right)^{-\mathrm{m}}$ gives Equation 10 . The probability of bit error of PRake receivers for EC-PPM modulation with a suboptimal template in the presence of timing- jitter is as given in Equation 11. The MGF for correlated Nakagami-m fading is (Simon and Alouni, 2000):

$M_{\bar{\eta}}(s)=\prod_{1=1}^{L}\left(\frac{1-s \bar{\eta}_{1}}{m L}\right)^{-m}\left[\operatorname{det}\left[c_{u j}\right]\right]^{-m}$

Where:

$c_{u j}=\left\{\begin{array}{cc}1 & u=j \\ \sqrt{\rho_{u j}}\left(1-\frac{m L}{s \bar{\eta}_{j}}\right)^{-1} & \text { o.w. }\end{array}\right.$

where, $\rho_{\mathrm{uj}}$ is the fading power correlation between subbands $\mathrm{u}$ and $\mathrm{j}$ (Simon and Alouni, 2000). The corresponding error probability is as Equation 15, where Equation (12):

$$
c_{\mathrm{uj}}=\left\{\begin{array}{cr}
1 & \mathrm{u}=\mathrm{j} \\
\sqrt{\rho_{\mathrm{uj}}}\left(1+\frac{\left(\mathrm{R}_{\mathrm{pv}}(0)-\mathrm{R}_{\mathrm{pv}}\left(\mu_{\mathrm{opt}}\right)\right) 4 \mathrm{~m}^{2} \mathrm{~L}_{\mathrm{p}} \sin ^{2} \theta}{\left(\mathrm{R}_{\mathrm{pv}}\left(\tau_{\mathrm{e}}\right)-\mathrm{R}_{\mathrm{pv}}\left(\mu_{\mathrm{opt}}-\tau_{\mathrm{e}}\right)\right)^{2} \bar{\eta}_{\mathrm{j}}}\right)^{-1} \quad \text { o.w. }
\end{array}\right.
$$

\subsection{Actual Measurements and Numerical Results}

In this section, we use the analysis provided in Section 3 to compare the performance of IR-UWB correlation receivers with optimal and suboptimal templates in AWGN and Nakagami-m channels in the presence of timing-jitter. We also provide results based on semi-analytic simulations in the IEEE 801.5.6a onbody CM\#3 UWB channel and actual on-body measurements. For our simulations, we assume the different Gaussian pulses with parameters that comply with the FCC indoor and outdoor masks.

As was previously mentioned, windowed-suboptimal template pulses have been proposed in the literature as alternatives to optimal Gaussian pulses. Figure 1 shows the optimal and suboptimal templates assuming the eighth order Gaussian pulse Equation (13 and 14):

$$
\mathrm{P}_{\mathrm{b}, \text { PRake }}=\frac{1}{\pi} \int_{0}^{\pi / 2}\left(\frac{\left(\mathrm{R}_{\mathrm{pp}}(0)-\mathrm{R}_{\mathrm{pp}}\left(\delta_{\mathrm{opt}}\right)\right) 4 \mathrm{~m} \sin ^{2} \theta}{\left(\mathrm{R}_{\mathrm{pp}}(0)-\mathrm{R}_{\mathrm{pp}}\left(\delta_{\mathrm{opt}}\right)\right) 4 \mathrm{~m} \sin ^{2} \theta+\bar{\eta}\left(\mathrm{R}_{\mathrm{pp}}\left(\tau_{\mathrm{e}}\right)-\mathrm{R}_{\mathrm{pp}}\left(\delta_{\mathrm{opt}}-\tau_{\mathrm{e}}\right)\right)^{2}}\right)^{\mathrm{mL} \mathrm{p}} \mathrm{d} \theta
$$




$$
\begin{aligned}
& \mathrm{P}_{\mathrm{b}, \text { PRake }}=\frac{1}{\pi} \int_{0}^{\pi / 2}\left(\frac{\left(\mathrm{R}_{\mathrm{pv}}(0)-\mathrm{R}_{\mathrm{pv}}\left(\mu_{\mathrm{opt}}\right)\right) 4 \mathrm{~m} \sin ^{2} \theta}{\left(\mathrm{R}_{\mathrm{pv}}(0)-\mathrm{R}_{\mathrm{pv}}\left(\mu_{\mathrm{opt}}\right)\right) 4 \mathrm{~m} \sin ^{2} \theta+\bar{\eta}\left(\mathrm{R}_{\mathrm{pv}}\left(\tau_{\mathrm{e}}\right)-\mathrm{R}_{\mathrm{pv}}\left(\mu_{\mathrm{opt}}-\tau_{\mathrm{e}}\right)\right)^{2}}\right)^{\mathrm{mL} \mathrm{p}} \mathrm{d} \theta \\
& \mathrm{P}_{\mathrm{b}, \text { PRake }}=\frac{1}{\pi} \int_{0}^{\pi / 2} \prod_{0}^{\mathrm{L}_{\mathrm{p}}}\left(\frac{\left(\mathrm{R}_{\mathrm{pv}}(0)-\mathrm{R}_{\mathrm{pv}}\left(\mu_{\mathrm{opt}}\right)\right) 4 \mathrm{~m} \sin ^{2} \theta+\bar{\eta}\left(\mathrm{R}_{\mathrm{pv}}\left(\tau_{\mathrm{e}}\right)-\mathrm{R}_{\mathrm{pv}}\left(\mu_{\mathrm{opt}}-\tau_{\mathrm{e}}\right)\right)^{2}}{4 \mathrm{~m}^{2} \mathrm{~L}_{\mathrm{p}} \sin ^{2} \theta\left(\mathrm{R}_{\mathrm{pv}}(0)-\mathrm{R}_{\mathrm{pv}}\left(\mu_{\mathrm{opt}}\right)\right)}\right)^{-\mathrm{m}}\left[\operatorname{det}\left[\mathrm{c}_{\mathrm{uj}}\right]\right]^{-\mathrm{m}} \mathrm{d} \theta
\end{aligned}
$$

The corresponding autocorrelation and crosscorrelation functions are shown in Fig. 2. As can be seen, the cross-correlation function resembles the autocorrelation function, which consequently leads to an approaching performance.

Typically, the normalized cross-correlation coefficient can be $>0.9$ for appropriate choice of the pulse parameters. However, this solution suffers from the sensitivity of the correlator output Signal-to-Noise Ratio (SNR) to timingerrors. It is worth noting that receiver structures with suboptimal templates are more sensitive to timing-errors as compared to optimal receivers (Sangyoub, 2002). Nevertheless, with the appropriate choice of the pulse parameters this drawback can be minimized. Figure 3 shows the BER of PRake receivers in independent Nakagami-m channels for two and four fingers and different values of timing-jitter values. Figure 4 shows the BER performance of EC-BPPM in the IEEE 802.15.6a on-body CM\#3 UWB channel for different timing-jitter values. Figure 5 shows the corresponding data-throughput also for different values of timing-jitter.

In order to evaluate the performance of the studied receiver for WBANs based on realistic data, actual measurements were taken at the Wireless at Virginia Tech research group labs. The following equipments were used: HP33120A function generator, TektronixCSA8000B Digital Sampling Oscilloscope, Geozondas pulser (GZ1106DL1, GZ1117DN-25) and antennas shown in Fig. 6. In the measurement setup, transmit and receive antennas having Line-of-Sight (LOS) link were attached to the test subject's body and the subject was allowed to move. The received pulses were recorded and stored with pulse length $=4000$ samples.

The normalized reference pulse is depicted in Fig. 7. A sample received pulse along with the corresponding suboptimal sinusoidal template is shown in Fig. 8. The corresponding autocorrelation and cross-correlation functions are depicted in Fig. 9. The resulting BER comparison between optimal and suboptimal template based detectors is shown in Fig. 10 for different values on timing-jitter. According to the figure, suboptimal templates are traded for a negligible BER performance degradation.
The corresponding maximum allowable data throughputs for optimal and suboptimal template based detectors are shown in Fig. 11 and 12, respectively. According to theoretical, simulation and measurement results, we can see that at a distance $\leq 10 \mathrm{~m}$, data throughout approaches 70 Mbps. The maximum SNR degradation is $\approx 2 \mathrm{~dB}$ and corresponding throughput penalty is $5 \mathrm{Mbps}$.

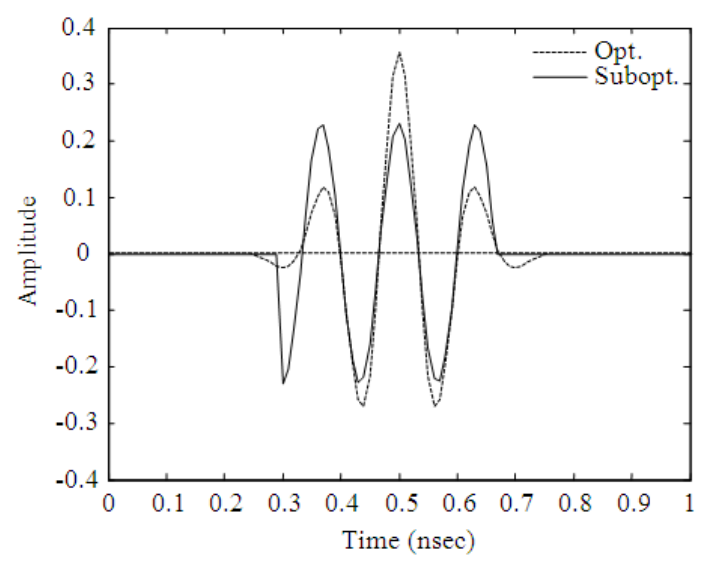

Fig. 1. Eighth order Gaussian pulse and corresponding sinusoidal template

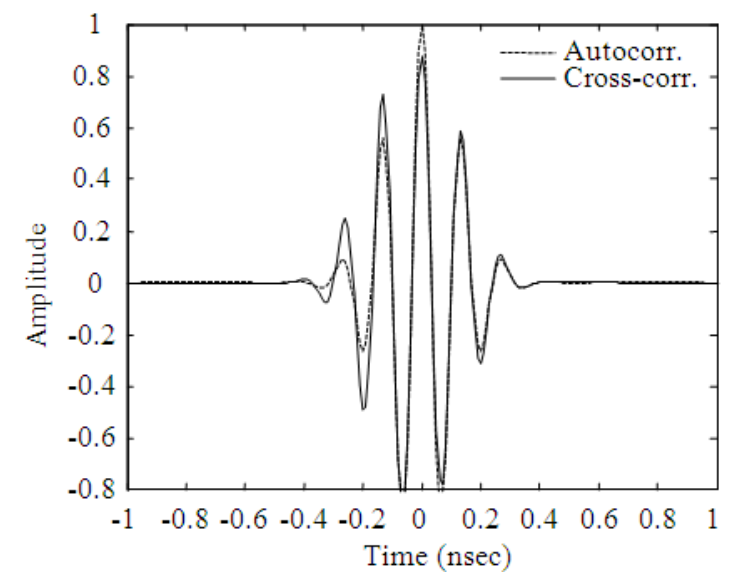

Fig. 2. Autocorrelation and cross-correlation functions 


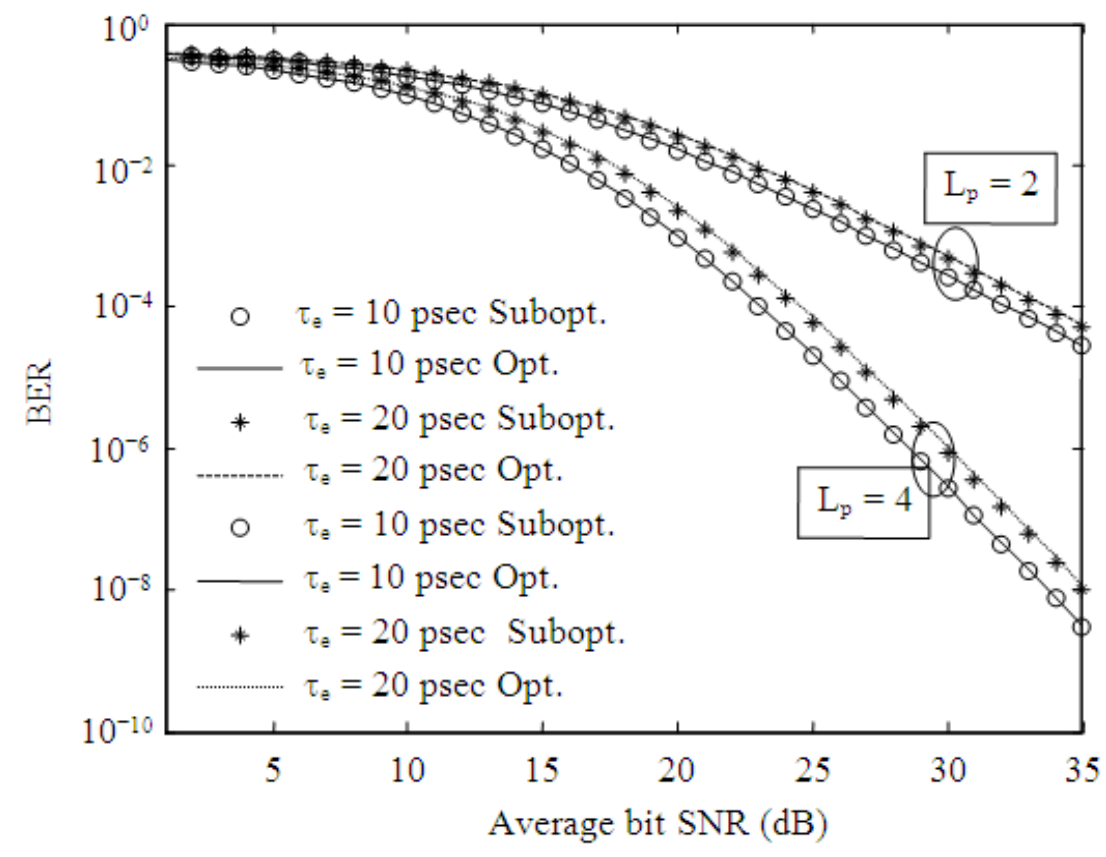

Fig. 3. BER performance comparison of PRake receivers for M-ary equally-correlated pulse position modulation (EC-PPM) scheme in independent Nakagami-m fading channels with uniform PDP, assuming different values of $\mathrm{L}_{\mathrm{p}}$ and $\mathrm{M}$, for the fifth order Gaussian pulse with optimal and suboptimal templates

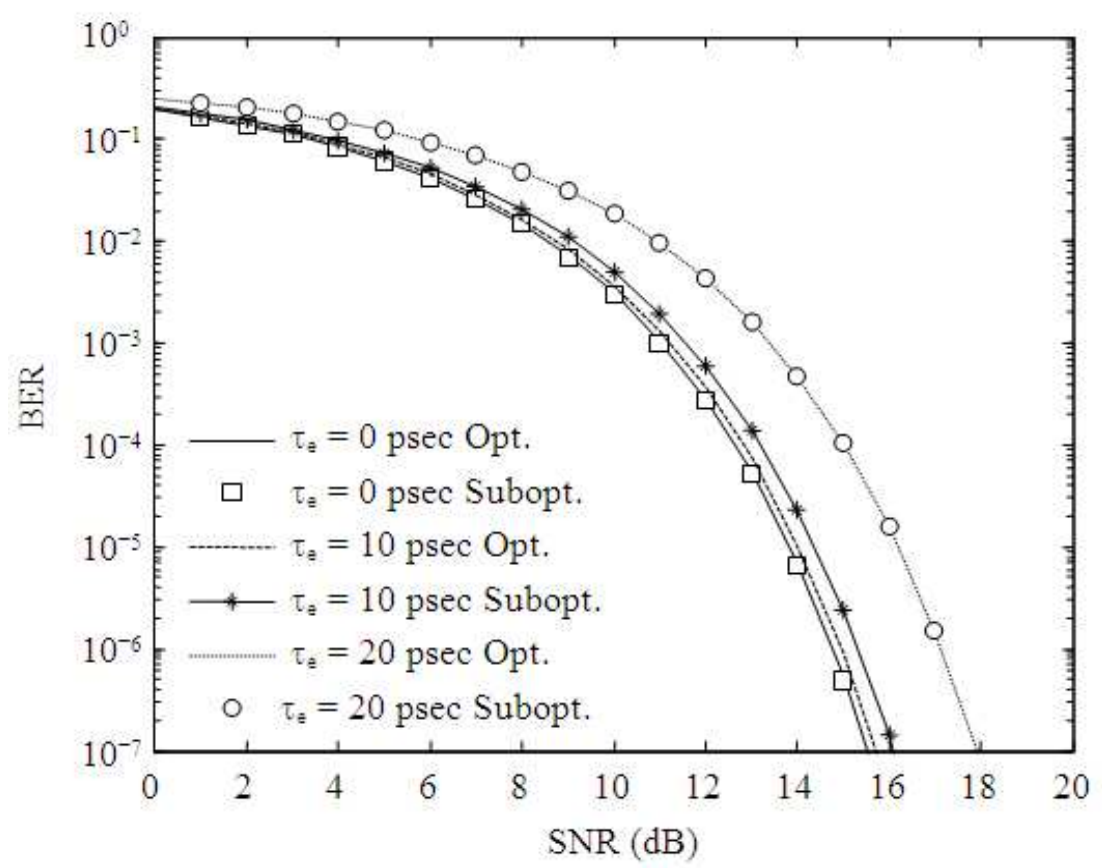

Fig. 4. BER performance comparison of EC-BPPM mod-ulation in IEEE 802.15.6a channel CM \#3 for the second order of the Gaussian pulse with optimal and suboptimal templates 
Mohamad Abou El-Nasr and Heba Shaban / American Journal of Applied Sciences 10 (5): 487-496, 2013

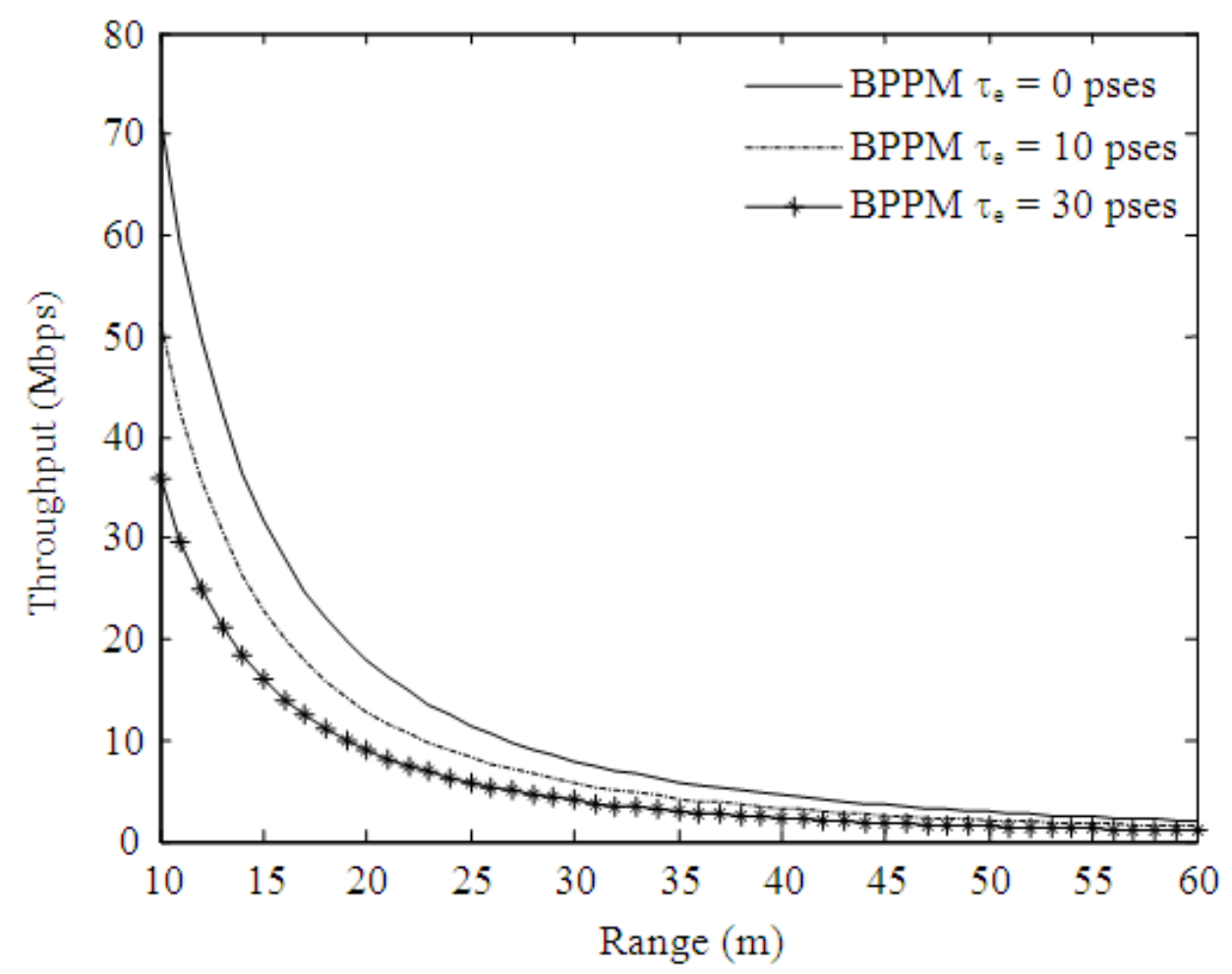

Fig. 5. Data throughput comparison of EC-BPPM modulation in IEEE 802.15.6a channel CM\#3 for the second order of the Gaussian pulse with optimal and suboptimal templates for different values of $\tau_{\mathrm{e}}$

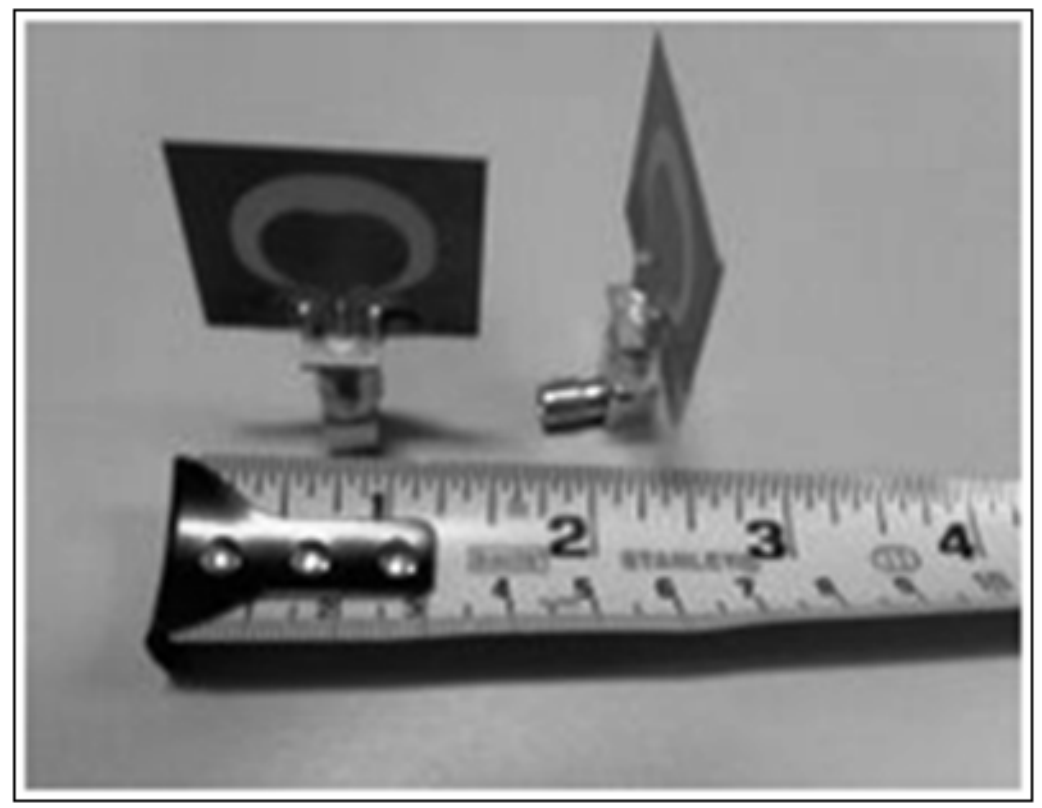

Fig. 6. UWB antennas manufactured by the Virginia Tech Antenna Group (VTAG) 
Mohamad Abou El-Nasr and Heba Shaban / American Journal of Applied Sciences 10 (5): 487-496, 2013

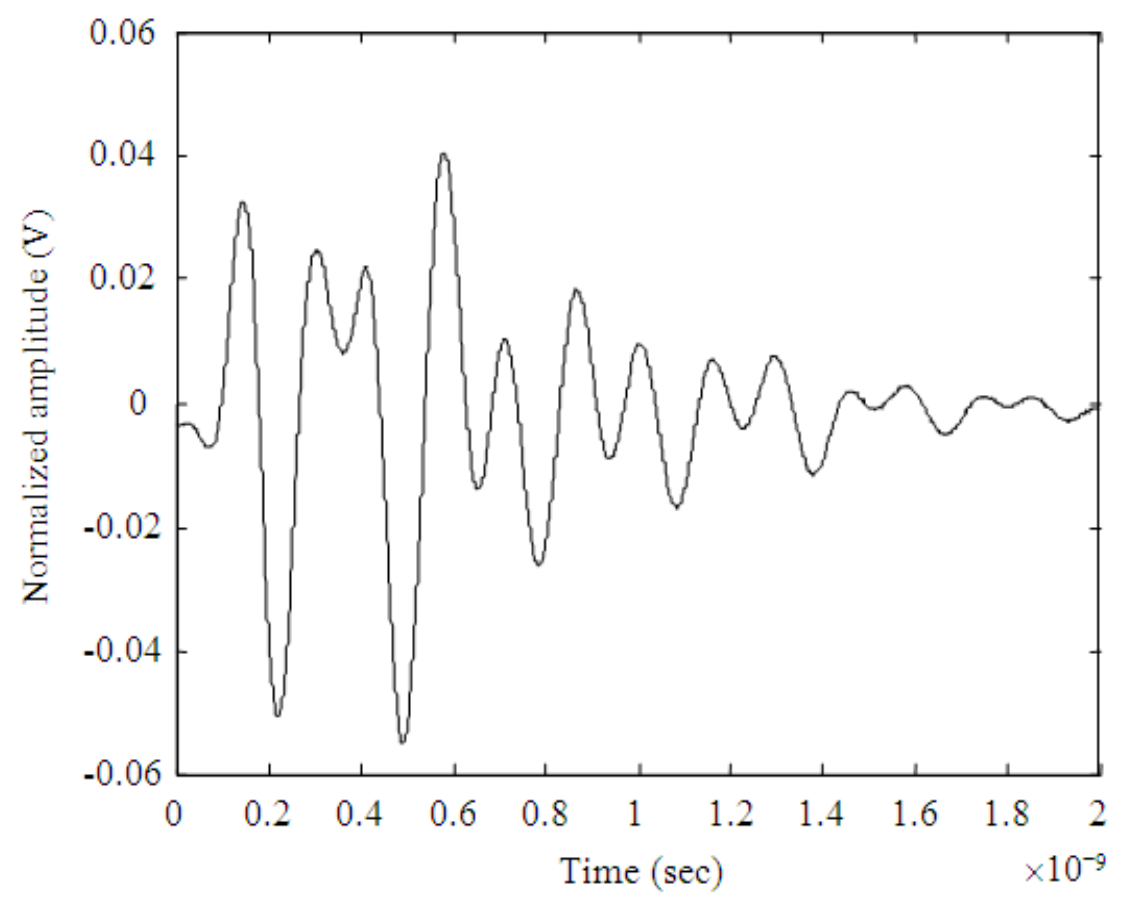

Fig. 7. Normalized measured reference pulse

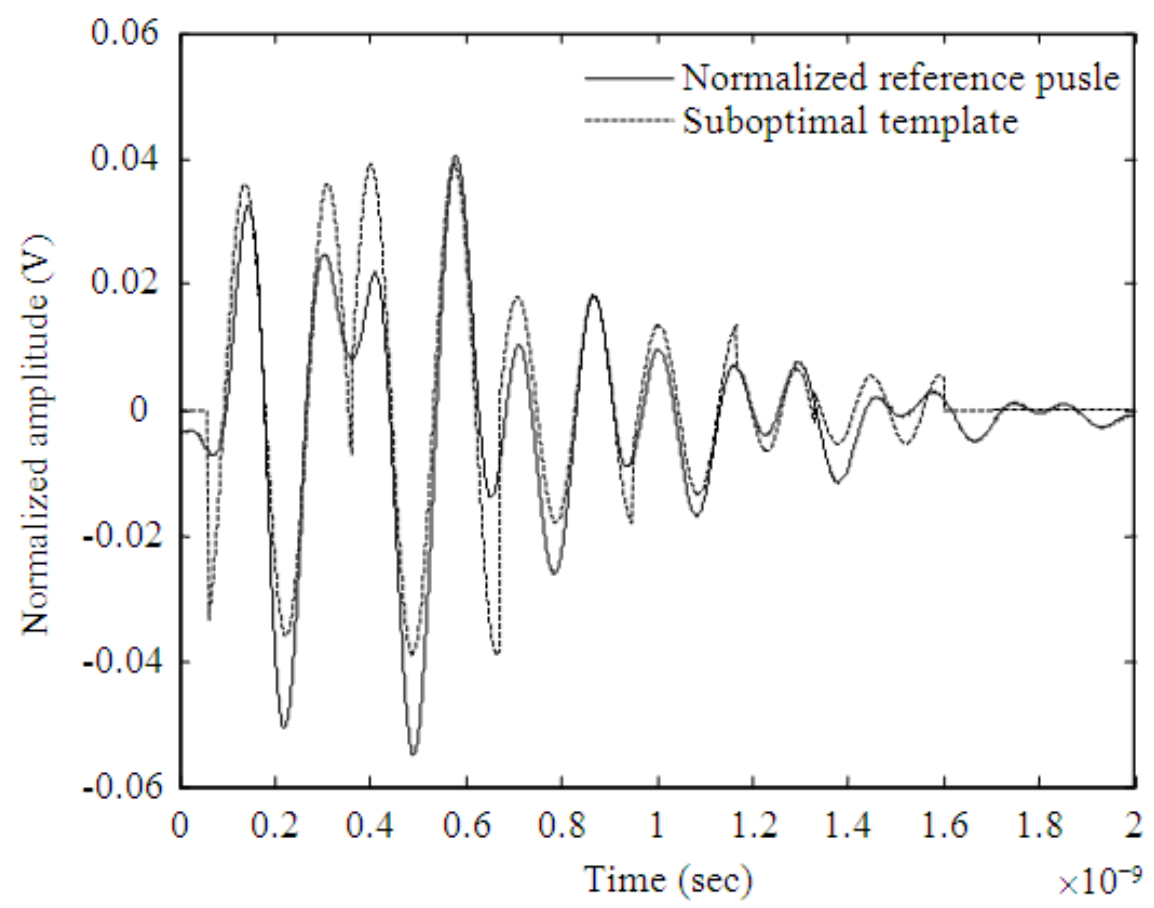

Fig. 8. Sample received pulse from actual measurements along with the sinusoidal template 
Mohamad Abou El-Nasr and Heba Shaban / American Journal of Applied Sciences 10 (5): 487-496, 2013

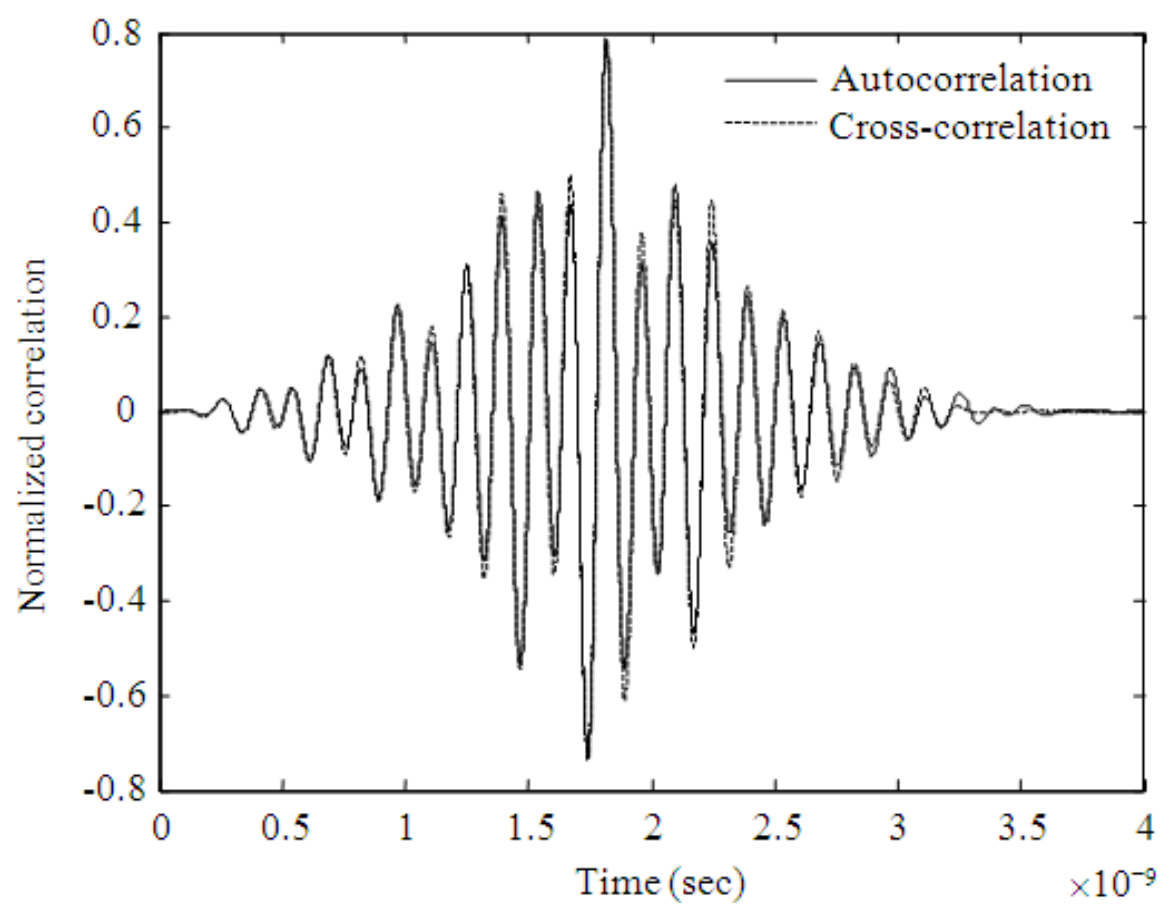

Fig. 9. Corresponding autocorrelation and cross-correlation functions

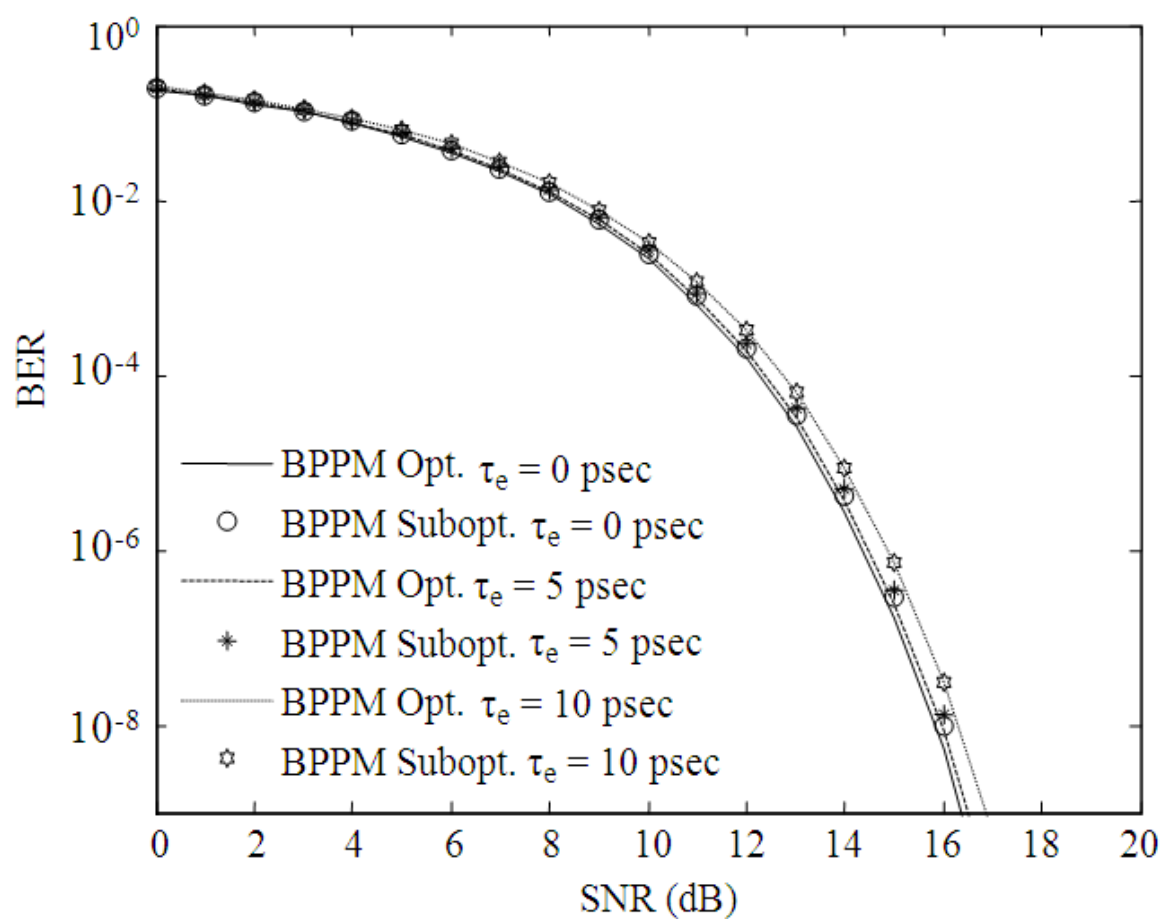

Fig. 10. BER performance comparison of EC-BPPM modulation based on actual measurements for $\tau_{\mathrm{e}}=0,5$ and $10 \mathrm{psec}$ 
Mohamad Abou El-Nasr and Heba Shaban / American Journal of Applied Sciences 10 (5): 487-496, 2013

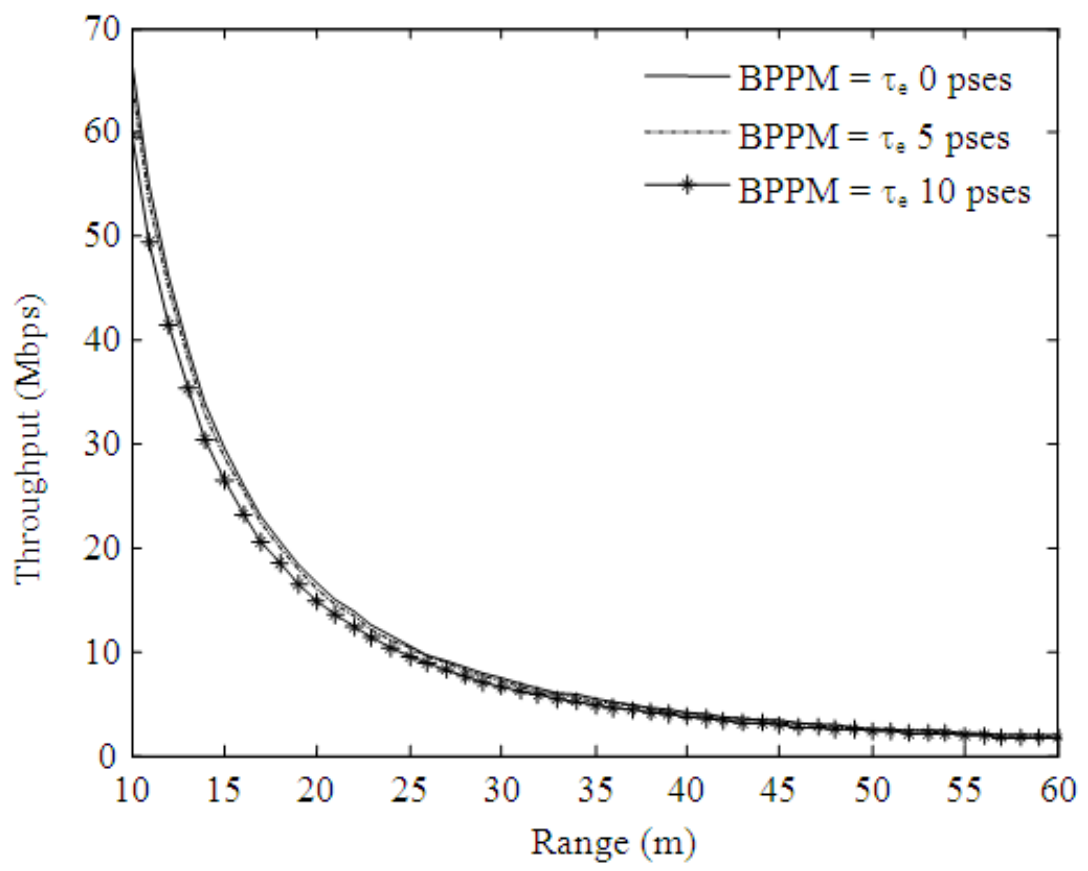

Fig. 11. Data throughput comparison of EC-BPPM modulation based on actual measurements for $\tau_{\mathrm{e}}=0,5$ and 10 psec with optimal template

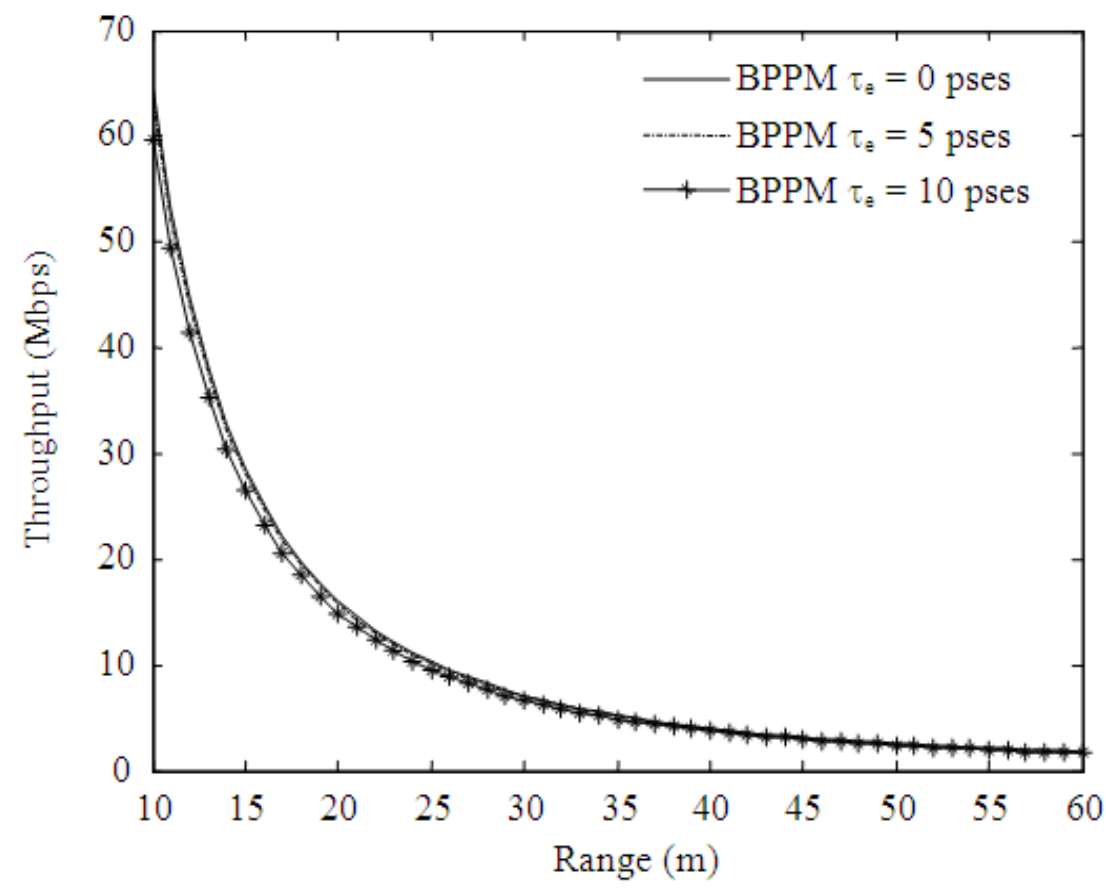

Fig. 12. Data throughput comparison of EC-BPPM modulation based on actual measurements for $\tau_{\mathrm{e}}=0,5$ and $10 \mathrm{psec}$ with suboptimal sinusoidal template 
Table 1. Data-rates for physiological data monitoring (Chavez-Santiago et al., 2009; Takizawa et al., 2007)

\begin{tabular}{ll}
\hline Biomedical measurement & Data rate \\
\hline Electrocardiogram (ECG) & $120 \mathrm{kbps}$ \\
Electromyography (EMG) & up to $1500 \mathrm{kbps}$ \\
Heart beat rate & $128 \mathrm{bps}$ \\
Oxygen saturation & $80 \mathrm{bps}$ \\
Blood pressure & $128 \mathrm{bps}$ \\
Body temperature & $80 \mathrm{bps}$ \\
Real-time video & $\approx 70 \mathrm{Mbps}$ \\
\hline
\end{tabular}

Generally, the low-power consumption of the studied receivers makes them attractive candidates for medical WBAN applications. In addition, as can be seen from the data-rate results, the achievable data throughput (even in the presence of timing-jitter) is on the order of tens of Mbps, which makes them suitable for the transmission for different types of medical signals, as depicted in Table $\mathbf{1 .}$

\section{CONCLUSION}

Windowed sinusoidal template-based IR-UWB correlation receivers are attractive candidates for high data-rate and high performance WBAN applications. Their performance approaches the performance of optimal receivers with minimal BER and data throughput degradation. The performance of a IR-UWB correlation receiver with a suboptimal sinusoidal template was analyzed and compared to the optimum receiver for ECPPM modulation in the presence of timing-jitter. Numerical results based on theoretical analysis, simulations as well as actual measurements showed that the BER performance degradation is $2 \mathrm{~dB}$ as compared to the optimum receiver and the corresponding degradation in data throughput is $5 \mathrm{Mbps}$.

\section{REFERENCES}

Chavez-Santiago, R., A. Khaleghi, I. Balasingham and T. Ramstad, 2009. Architecture of an ultra wideband wireless body area network for medical applications. Proceedings of 2nd International Symposium on Applied Sciences in Biomedical and Communication Technologies, Nov. 24-27, IEEE Xplore Press, Norway, pp: 1-6. DOI: 10.1109/ISABEL.2009.5373624

Guvenc, I. and H. Arslan, 2004. Performance analysis of UWB systems in the presence of timing jitter. J. Commun. Netw., 6: 182-191.

$\mathrm{Hu}$, B. and N.C. Bueaulieu, 2005. Pulse shapes for ultra wideband communication systems. IEEE Trans. Wireless Commun., 4: 1789-1797. DOI: 10.1109/TWC.2005.850311
Lazaro, A., D. Girbau and R. Villarin, 2010. Analysis of vital signs monitoring using an IR-UWB radar. Progress Electromagnet. Res., 100: 265-284. DOI: 10.2528/PIER09120302

Quek, T.Q.S. and M.Win, 2005. Analysis of UWB transmitted-reference communication systems in dense multipath channels. IEEE J. Selected Areas Commun., 23: 1863-1874. DOI: 10.1109/JSAC.2005.853809

Remirez-Mireles, F., 2001. On the performance of ultra wide-band signals in Gaussian noise and dense multipath. IEEE Trans. Vehicular Technol., 5: 244249. DOI: $10.1109 / 25.917932$

Sangyoub, L., 2002. Design and analysis of ultra-wide bandwidth impulse radio receiver. Ph.D. Thesis, Southern California University, California, USA.

Shaban, H., 2010. A novel highly accurate wireless wearable human locomotion tracking and gait analysis system via UWB radios. Ph.D. Thesis, Virginia Tech, Virginia, USA.

Shaban, H., M.A. El-Nasr and R. Buehrer, 2009. Receivers for highly accurate wearable human locomotion tracking and gait analysis systems. Proceedings of IEEE Global Telecommunications Conference, Nov. 30-Dec. 4, IEEE Xplore Press, USA., pp: 1-6. DOI: 10.1109/GLOCOM.2009.5426230

Shaban, H., M.A. El-Nasr and R. Buehrer, 2010. Toward a highly accurate ambulatory system for clinical gait analysis via UWB radios. IEEE Trans. Inform. Technol. Biomed., 14: 284-291. DOI: 10.1109/TITB.2009.2037619

Shaban, H., M.A. El-Nasr and R. Buehrer, 2011. Reference Range correlation (RRcR) ranging and performance bounds for on-body UWB-based body sensor networks. Progress Electromagnet. Res. B, 35: 69-85. DOI: 10.2528/PIERB1 1082212

Simon, M.K. and K.S. Alouni, 2000. Digital Communication over Fading Channels: A Unified Approach to Performance Analysis. 1st Edn., Wiley, New York, ISBN-10: 0471317799.

Takizawa, K., H.B. Li, K. Hamaguchi and R. Kohno, 2007. Wireless vital sign monitoring using ultra wideband-based personal area networks. Proceedings of 29th Annual International Conference of the IEEE EMB, Aug. 22-26. IEEE Xplore Press, France, pp: 1798-1801. DOI: 10.1109/IEMBS.2007.4352661

Yazdandoost, K.Y. and K.S. Pour, 2010. Channel model for Body Area Network (BAN). Technical Report of IEEE P802.15-08-0780-10-0006, IEEE P802.15 Working Group for Wireless Personal Area Networks (WPANs), USA. 